\title{
MULTIVARIATE NEWTON SUMS: IDENTITIES AND GENERATING FUNCTIONS
}

\section{Emmanuel Briand $^{1}$ and Laureano Gonzalez-Vega ${ }^{2}$}

\author{
${ }^{1}$ IRMAR, Université de Rennes I Rennes, France \\ E-mail: ebriand@maths.univrennes1.fr \\ ${ }^{2}$ Dpto. Matemáticas, Estadística y Computación Universidad de Cantabria, \\ Santander, Spain \\ E-mail: gvega@matesco.unican.es
}

\begin{abstract}
This paper is devoted to present, first, a family of formulas extending to the multivariate case the classical Newton (or Newton-Girard) Identities relating the coefficients of a univariate polynomial equation with its roots through the Newton Sums and, secondly, the Generating Functions associated to the new introduced Newton Sums of the mul-tivariate case. As a by-product the kinds of systems accepting these Newton Identities are also characterized together with those allowing the Newton Sums to be computed in an inductive way directly from the coefficients of the polynomial system under consideration.
\end{abstract}




\section{INTRODUCTION}

When considering a univariate polynomial with coefficients in a field $\mathbb{K}$ with characteristic 0 , its Newton Sums and its coefficients are connected by the well-known Newton Identities. Dealing with $n$ polynomials in $n$ unknowns with coefficients in a subfield $\mathbb{K}$ of the field of the complex numbers $\mathrm{C}$, and finitely many solutions in $\mathbb{C}^{n},^{[1]}$ showed that there exists a generalization of these formulas (Multivariate Newton Identities). They also pointed out that, when the system was a Pham System, i-e had the shape:

$$
F_{i}=X_{i}^{d_{i}}+\text { terms with total degree } \leq d_{i}-1 \quad(1 \leq i \leq n),
$$

then these identities made possible the computation of the Multivariate Newton Sums from the coefficients, by an induction procedure, as in the univariate case.

In a previous paper ${ }^{[8]}$ it was shown how to obtain the same results with the algebraic version of the global residue operator (which made the method valid for systems with coefficients in any field $\mathbb{K}$ with characteristic 0 ). These results can be extended, in an algebraic way too, to the so-called Pham systems of type II (see also the analytic version $\mathrm{in}^{[1]}$ ).

In this paper we show that, using the same techniques introduced by ${ }^{[8]}$, the results extend to the case of Generalized Pham Systems, i-e systems:

$$
F_{i}=X_{i}^{d_{i}}+\text { lower terms w.r.t. } \preceq \quad(1 \leq i \leq n)
$$

for some term ordering $\preceq$. This precisely means that, besides defining a zerodimensional complete intersection, the $F_{i}$ 's are a Grobner basis for $\preceq$ (see for instance ${ }^{[12]}$, Lemma 6.15$)$. The vector $\delta=\left(d_{1}, \ldots, d_{n}\right)$ is called the multidegree of the Generalized Pham System.

In the first part we present the elements of the algebraic theory of the global residue operator to be used in the sequel. We also prove that when $F$ is a Generalized Pham System, the corresponding global residue operator maps exactly a polynomial on the leading coefficient of its normal form. This was already demonstrated in ${ }^{[5]}$, but our proof is direct. Besides, it leads naturally to a new proof of the Euler-Jacobi Theorem and Macaulay Theorem.

Next we derive the Multivariate Newton Identities for Generalized Pham Systems $F=\left(F_{1}, \ldots, F_{n}\right)$ and show how to use them as induction formulas to relate the generalized Newton sums (being defined most of them as the traces of monomials) with the coefficients of the polynomials $F_{i}$ 's.

The third part focuses on presenting the generating function for the generalized Newton sums introduced in the second section: $i-e$ a rational 
function in the variables $X_{1}, \ldots, X_{n}$ whose expansion in negative powers of the variables provides the generalized Newton sums. This result is generalized to the case where, instead of the generalized Newton sums, we generate the image of the monomials under any linear form defined in the quotient $\mathbb{K}[\underline{X}] /\left\langle F_{1}, \ldots, F_{n}\right\rangle$. This answers in a positive way a question posed by E. Becker.

The last section shows how the previously mentioned Generating Functions for the Multivariate Newton Sums provide explicit formulas for the trace of some monomials with respect to a Pham System of degree 2.

\section{ALGEBRAIC PRELIMINARIES}

Let $\mathbb{K}$ be a field of characteristic 0 and $\mathbb{L}$ an algebraically closed field containing $\mathbb{K}$. Let $F_{1}, \ldots, F_{n}$ be polynomials in $\mathbb{K}[\underline{X}]=\mathbb{K}\left[X_{1}, \ldots, X_{n}\right]$. We assume the ideal they generate, $J$, is a zero-dimensional complete intersection. Equivalently: the quotient algebra $\mathcal{A}=\mathbb{K}[\underline{X}] / J$ is finite-dimensional over $\mathbb{K}$. We denote by $\Delta_{1}, \ldots, \Delta_{r}$ the roots of $J$ in $\mathbb{L}^{n}$, and $\mu_{1}, \ldots, \mu_{r}$ their respective multiplicities. Then $\mathcal{A}$ has dimension $D=\sum \mu_{i}$. This section focuses on the explicit presentation of some algebraic properties of $\mathcal{A}$ to be used in the sequel.

\subsection{The Dualizing Linear Form}

Since the ideal $J$ is a zero dimensional complete intersection, $\mathcal{A}$ is a Frobenius Algebra ${ }^{[4]}$ : there exists a linear form $\phi: \mathcal{A} \rightarrow \mathbb{K}$ (called a dualizing linear form) such that the symmetric $\mathbb{K}$-bilinear form

$$
\begin{aligned}
\mathcal{A} \times \mathcal{A} & \rightarrow \mathbb{K} \\
(a, b) & \mapsto \phi(a b)
\end{aligned}
$$

is non degenerate. [4] shows how to find such a dualizing linear form on $\mathcal{A}$. We recall it now.

For $P \in \mathbb{K}[\underline{X}]$ and $1 \leq i \leq n$ we define $\partial_{i}(P)\left(X_{1}, \ldots, X_{n}, Y_{1}, \ldots, Y_{n}\right)=$ $\partial_{i}(P)(\underline{X}, \underline{Y})$ as follows:

$$
\partial_{i}(P)(\underline{X}, \underline{Y})=\frac{P\left(Y_{1}, \ldots, Y_{i-1}, X_{i}, \ldots, X_{n}\right)-P\left(Y_{1}, \ldots, Y_{i}, X_{i+1}, \ldots, X_{n}\right)}{X_{i}-Y_{i}}
$$

Then $\operatorname{Bez}(\underline{X}, \underline{Y})$, the Bezoutian Determinant associated to $F_{1}, \ldots, F_{n}$, is the determinant of the matrix of the $\partial_{j}\left(F_{i}\right)$ for $1 \leq i, j \leq n$ (it is a polynomial in $\mathbb{K}[\underline{X}, \underline{Y}])$. 
Let $\mathcal{A} \otimes_{\mathbb{K}} \mathcal{A}$ be the quotient $\mathbb{K}[\underline{X}, \underline{Y}] /\left\langle F_{1}(\underline{X}), \ldots, F_{n}(\underline{X}), F_{1}(\underline{Y}), \ldots\right.$, $\left.F_{n}(\underline{Y})\right\rangle$ and $x_{i}$ and $y_{i}$ the images of $X_{i}$ and $Y_{i}$ in $\mathcal{A} \otimes_{\mathbb{K}} \mathcal{A}$. So $\operatorname{Bez}(\underline{x}, \underline{y})$ is the image in $\mathcal{A} \otimes_{\mathbb{K}} \mathcal{A}$ of $\operatorname{Bez}(\underline{X}, \underline{Y})$.

If $\mathcal{B}=\left\{\varepsilon_{i}(\underline{x}): 1 \leq i \leq D\right\}$ is a basis of the $\mathbb{K}$-algebra $\mathcal{A}$ then there exists a decomposition:

$$
\operatorname{Bez}(\underline{x}, \underline{y})=\widehat{\varepsilon_{1}}(\underline{y}) \varepsilon_{1}(\underline{x})+\cdots+\widehat{\varepsilon_{D}}(\underline{y}) \varepsilon_{D}(\underline{x}) .
$$

Then $\hat{\mathcal{B}}=\left\{\widehat{\varepsilon}_{i}(\underline{x}): 1 \leq i \leq D\right\}$ happens to be also a basis of $\mathcal{A}$ (the dual basis of $\mathcal{B}$ ), and given the decomposition of 1 in $\mathcal{A}$ according to the dual basis $\widehat{\mathcal{B}}$, $1=d_{1} \widehat{\varepsilon_{1}}(\underline{x})+\cdots+d_{D} \widehat{\varepsilon_{D}}(\underline{x})$, the linear form on $\mathcal{A}$ defined by $\varepsilon_{i}(\underline{x}) \mapsto d_{i}$ is dualizing. This form $\ell_{F}=\ell_{\left[F_{1}, \ldots, F_{n}\right]}$ is called the Kronecker Symbol or global residue operator associated to $F_{1}, \ldots, F_{n}$. We will take the liberty of denoting also by $\ell_{F}$ the linear form it induces on $\mathbb{K}[\underline{X}]$, when convenient. When $\mathbb{K} \subset \mathbb{C}$, the Kronecker Symbol coincides with the classical residue (see ${ }^{[14]}$ and $\left.^{[5]},(\S 0)\right)$.

When $J$ is a radical ideal the global residue operator verifies:

$$
\ell_{F}(P)=\sum_{i=1}^{r} \frac{P\left(\Delta_{i}\right)}{\mathbf{J a c}\left(\Delta_{i}\right)}
$$

where Jac denotes the Jacobian determinant of the polynomials $F_{1}, \ldots, F_{n}$. Without assumptions on $J$ the dualizing form $\ell_{F}$ is related with the trace by the following equality:

$$
\ell_{F}(P \cdot \mathbf{J a c})=\mu_{1} P\left(\Delta_{1}\right)+\cdots+\mu_{r} P\left(\Delta_{r}\right)=\operatorname{Trace}(P) .
$$

A very useful tool to deal with the dualizing form is the Transformation Law which is presented in the following proposition $\left({ }^{[6]}\right.$ and ${ }^{[13]}$ give an algebraic proof of this classical result).

Proposition 1.1 (Transformation Law). Let $G_{1}, \ldots, G_{n}$ be polynomials in $\mathbb{K}[\underline{X}]$ with a finite number of solutions in $\mathbb{L}^{n}$ and $G_{i}=T_{i 1} F_{1}+\cdots+T_{i n} F_{n}$, $T_{i j} \in \mathbb{K}[\underline{X}](i \in\{1, \ldots, n\})$. If $P \in \mathbb{K}[\underline{X}]$ then $\ell_{F}(P)=\ell_{G}(P \cdot \operatorname{det}(T))$.

\subsection{Expression of the Dualizing Form for a Generalized Pham System}

Now we consider the special case when $F$ is a Generalized Pham System for some term ordering $\preceq$. The family of the $\varepsilon_{\alpha}(\underline{x})=x_{1}^{\alpha_{1}} \cdots \cdots x_{n}^{\alpha_{n}}=$ $\mathbf{x}^{\alpha}$, for $0 \leq \alpha_{1}<d_{1}, \ldots, 0 \leq \alpha_{n}<d_{n}$, is a basis over $\mathbb{K}$ of the quotient algebra. 
We notice that we can write, for $i \neq j, \partial_{j}\left(F_{i}\right)=b_{i, j}(\underline{X}, \underline{Y}) / X_{j}$ and

$$
\partial_{j}\left(F_{j}\right)=\left(X_{j}^{d_{j}}+b_{j, j}(\underline{X}, \underline{Y})\right) / X_{j}
$$

where the $b_{i, j}$ 's, seen as polynomials in $\underline{X}$ with coefficients in $\mathbb{K}[\underline{Y}]$, have all their monomials smaller than $X_{i}^{d_{i}}$ with respect to $\preceq$. Thus, if we develop the determinant defining the Bezoutian then we obtain:

$$
\operatorname{Bez}(\underline{X}, \underline{Y})=\frac{1}{X_{1} \cdots X_{n}} \cdot\left(X_{1}^{d_{1}} \cdots X_{n}^{d_{n}}+R(\underline{X}, \underline{Y})\right)
$$

with all $\underline{X}$-monomials in $R$ smaller than $X_{1}^{d_{1}} \cdots \cdots X_{n}^{d_{n}}$. Otherwise stated:

$$
\operatorname{Bez}(\underline{X}, \underline{Y})=X_{1}^{d_{1}-1} \cdots \cdots X_{n}^{d_{n}-1}+R_{1}(\underline{X}, \underline{Y})
$$

with all $\underline{X}$-monomials in $R_{1}$ smaller than $X_{1}^{d_{1}-1} \cdots X_{n}^{d_{n}-1}$.

The reduction of $R_{1}$ to its normal form with respect to $F_{1}(\underline{X}), \ldots$, $F_{n}(\underline{X}), F_{1}(\underline{Y}), \ldots, F_{n}(\underline{Y})$ never produces the monomial $X_{1}^{d_{1}-1} \cdots X_{n}^{d_{n}}-1$, so we get:

$$
\operatorname{Bez}(\underline{x}, \underline{y})=x_{1}^{d_{1}-1} \cdots \cdots x_{n}^{d_{n}-1}+\sum_{\beta_{i}<d_{i}, i=1 \ldots n} \mathbf{x}^{\beta} \hat{\varepsilon}_{\beta}(\mathbf{y}) .
$$

Especially, $\hat{\varepsilon}_{\left(d_{1}-1, \ldots, d_{n}-1\right)}(\underline{y})=1$, whence, for $0 \leq \alpha_{1}<d_{1}, \ldots, 0 \leq \alpha_{n}<d_{n}$ :

$$
\ell_{\left[F_{1}, \ldots, F_{n}\right]}\left(\mathbf{x}^{\alpha}\right)= \begin{cases}1 & \text { if } \alpha=\left(d_{1}-1, \ldots, d_{n}-1\right) . \\ 0 & \text { otherwise }\end{cases}
$$

We have just proved the following result which, for $\mathbb{K} \subset \mathbb{C}$, is Lemma 4.2 of $^{[5]}$.

Proposition 1.2. Let $\left(F_{1}, \ldots, F_{n}\right)$ be a Generalized Pham System for a term ordering $\preceq$, and $X_{i}^{d_{i}}$ the leading term of every $F_{i}$. Then $\ell_{F}(P)$ is exactly the coefficient of $X_{1}^{d_{1}-1} \cdots \cdots X_{n}^{d_{n}-1}$ in the normal form of $P \in \mathbb{K}[\underline{X}]$ with respect to $F_{1}, \ldots, F_{n}$ and $\preceq$.

\subsection{Euler-Jacobi Theorem and Macaulay's Theorem}

For $H \in \mathbb{K}[\underline{X}]$, its Newton Diagram $\mathcal{N D}(H) \subset Z^{n}$ is the set of the exponents of the monomials in $H$ with non-zero coefficient. The Newton Polytope of $H$, denoted with $\mathcal{N P}(H)$, is the convex hull of $\mathcal{N D}(H)$ in $\mathbb{R}^{n}$. 
The Newton Polytope of the product $F_{1} \cdots F_{n}$ is also the Minkowski sum of the $\mathcal{N} \mathcal{P}\left(F_{i}\right)$ 's:

$$
\mathcal{N} \mathcal{P}\left(F_{1} \cdots F_{n}\right)=\mathcal{N} \mathcal{P}\left(F_{1}\right)+\cdots+\mathcal{N} \mathcal{P}\left(F_{n}\right) .
$$

For $E \subset Z^{n}$, we denote the semi-group generated by $E$ (the set of all the finite sums $e_{1}+\cdots+e_{m}$ with the $e_{i} \in E$ ) with $\sum E$.

We begin by looking at the special case when $\left(F_{1}, \ldots, F_{n}\right)$ is a Generalized Pham System, with $X_{1}^{d_{1}}, \ldots, X_{n}^{d_{n}}$ the respective leading terms of $F_{1}, \ldots, F_{n}$, and $\delta=\left(d_{1}, \ldots, d_{n}\right)$. Let $\mathcal{S}(F)$ be the semi-group generated by the elements of the set $\left\{\delta+\alpha \mid \alpha \in \cup_{i=1}^{n} \mathcal{N} \mathcal{D}\left(F_{i}\right)\right\}$. This set $\mathcal{S}(F)$ is always contained in

$$
\mathcal{C}(F)=\left\{\delta-t \cdot x: t \in \mathbb{R}^{+}, x \in \mathcal{N P}\left(F_{1} \cdots F_{n}\right)\right\}
$$

the polyhedron obtained by drawing half-lines from $\delta$ through all points of $\mathcal{N}\left(F_{1} \cdots F_{n}\right)$, next translating it by $-\delta$ and finally rotating it by half a turn around 0 . The origin 0 is a vertex for $\mathcal{C}$, and for any linear form $\mathbf{w}$ in the normal cone of $\mathcal{N} \mathcal{P}\left(F_{1} \cdots F_{n}\right)$ at $\delta$, the hyperplane $\mathbf{w}=0$ is a supporting hyperplane of $\mathcal{C}(F)$ at 0 . When all the $F_{i}$ 's coefficients are non-zero and have independent parameters then $\mathcal{S}(F)=\mathcal{C}(F) \cap Z^{n}$.

Proposition 1.3. Let $F$ be a Generalized Pham System. If $P \in \mathbb{K}[\underline{X}]$ and $\mathcal{N D}(P)$ lies outside $\{\delta-1\}+\mathcal{S}(F)$ then $\ell_{F}(P)=0$.

Here 1 is the vector $(1, \ldots, 1) \in \mathbb{Z}^{n}$.

Proof. Suppose $P$ reduces through $F_{1}$, for instance, into a polynomial $Q$. Then:

$$
\mathcal{N D}(Q) \subset \mathcal{N D}(P)+\mathcal{N D}\left(F_{1}\right)+\left\{\left(-d_{1}, 0, \ldots, 0\right)\right\}
$$

More generally, if $R$ is obtained from $P$ after a series of reductions by $F_{1}, \ldots, F_{n}$, we'll have:

$$
\begin{aligned}
& \mathcal{N D}(R) \subset \mathcal{N D}(P)+\sum\left(\mathcal{N D}\left(F_{1}\right)+\left\{\left(-d_{1}, 0, \ldots, 0\right)\right\}\right)+\cdots \\
& \quad+\sum\left(\mathcal{N D}\left(F_{n}\right)+\left\{\left(0, \ldots, 0,-d_{n}\right)\right\}\right)
\end{aligned}
$$

Note that actually:

$$
\begin{aligned}
& \sum\left(\mathcal{N D}\left(F_{1}\right)+\left\{\left(-d_{1}, 0, \ldots, 0\right)\right\}\right)+\cdots \\
& \quad+\sum\left(\mathcal{N D}\left(F_{n}\right)+\left\{\left(0, \ldots, 0,-d_{n}\right)\right\}\right)=-\mathcal{S}(F)
\end{aligned}
$$


for every set $\sum\left(\mathcal{N D}\left(F_{i}\right)+\left\{\left(\ldots, 0,-d_{i}, 0, \ldots\right)\right\}\right)$ containing $\{0\}$. After sufficiently many reductions, $R$ happens to be the normal form of $P$. Its global residue is non-zero if and only if its Newton Diagram contains $\{\delta-1\}$. This would involve that $\{\delta-1\} \in \mathcal{N D}(P)+(-\mathcal{S}(F))$ whence the proposition.

Example 1.1. A very special case is when the polynomials are pure powers: $F_{1}=X_{1}^{d_{1}}, \ldots, F_{n}=X_{n}^{d_{n}}$. Then the global residue operator acts on $\mathbb{K}[\underline{X}]$ as follows:

$$
\ell_{\left[X_{1}^{d_{1}}, \ldots, X_{n}^{d_{n}}\right]}\left(\mathbf{X}^{\alpha}\right)= \begin{cases}1 & \text { if } \alpha=\left(d_{1}-1, \ldots, d_{n}-1\right) \\ 0 & \text { otherwise }\end{cases}
$$

Let us introduce some vocabulary. Let $w \in \mathbb{N}^{n}$ (a weight vector). It determines a linear form on $\mathbb{R}^{n}$ : the weight $\mathbf{w}:\left(t_{1}, \ldots, t_{n}\right) \mapsto w_{1} t_{1}+\cdots$ $+w_{n} t_{n}$. The $\mathbf{w}$-degree of a monomial $\mathbf{X}^{\alpha}$ is defined as $\mathbf{w}(\alpha)$. The $\mathbf{w}$-degree of a non-zero polynomial $P$ is the integer $m=\operatorname{deg}_{w}(P)$ such that $P=H+R$ with all monomials in $H$ having $\mathbf{w}$-degree $m$, and all monomials in $R$ having w-degrees lower than $m$. The polynomial $H$ is then the w-homogeneous leading part of $P$. A root of $H$, if different from the trivial root $(0, \ldots, 0)$, is said to be a root at the weighted infinity, with respect to $\mathbf{w}$, for $P$.

If $F_{1}, \ldots, F_{n}$ have no common root at the weighted infinity with respect to $\mathbf{w}$, they are said to define a strict complete intersection graded by w. Strict complete intersections are studied in ${ }^{[10]}$ and in $^{[5]}$.

Example 1.2. A General Pham System $F$ defines a strict complete intersection, graded by any weight $\mathbf{w}$ lying inside the normal cone of $\mathcal{N} \mathcal{P}\left(F_{1} \ldots \ldots\right.$ $\left.F_{n}\right)$ at $\delta$, where $\delta$ is the multidegree of $F$.

Conversely, if the system $F=\left(F_{1}, \ldots, F_{n}\right)$ defines a strict complete intersection graded by $\mathbf{w}$ and such that the leading $\mathbf{w}$-homogeneous parts of $F_{1}, \ldots, F_{n}$ are pure powers, $X_{1}^{d_{1}}, \ldots, X_{n}^{d_{n}}$, then it is a Generalized Pham System for any term ordering $\preceq$ compatible with $\mathbf{w}$ in the following sense: $\mathbf{w}\left(\mathbf{X}^{\alpha}\right)<\mathbf{w}\left(\mathbf{X}^{\beta}\right) \Rightarrow \mathbf{X}^{\alpha} \prec \mathbf{X}^{\beta}$. One can construct such an ordering by choosing an arbitrary term ordering $\preceq_{T}$ and setting:

$$
\mathbf{X}^{\alpha} \preceq \mathbf{X}^{\beta} \Longleftrightarrow \mathbf{w}\left(\mathbf{X}^{\alpha}\right)<\mathbf{w}\left(\mathbf{X}^{\beta}\right) \quad \text { or }\left[\mathbf{w}\left(\mathbf{X}^{\alpha}\right)=\mathbf{w}\left(\mathbf{X}^{\beta}\right) \quad \text { and } \quad \mathbf{X}^{\alpha} \preceq_{T} \mathbf{X}^{\beta}\right]
$$

With a classical method and using the Transformation Law, the study of $\ell_{F}$ in the zero-dimensional strict complete intersection case is reduced to the case of Generalized Pham Systems. Suppose $F_{1}, \ldots, F_{n}$ define a zerodimensional strict complete intersection graded by $\mathbf{w}$. Let $H_{1}, \ldots, H_{n}$ be the 
respective w-homogeneous leading parts of $F_{1}, \ldots, F_{n}$. Then, for $\rho_{1}, \ldots, \rho_{n} \in$ $\mathbb{N}$ large enough, there exist polynomials $T_{i j}$ such that:

$$
X_{i}^{w_{i} \rho_{i}}=T_{i 1}\left(\underline{X}^{w}\right) \cdot H_{1}\left(\underline{X}^{w}\right)+\cdots+T_{i n}\left(\underline{X}^{w}\right) \cdot H_{n}\left(\underline{X}^{w}\right) \quad(1 \leq i \leq n)
$$

where $\underline{X}^{w}$ stands for $X_{1}^{w_{1}}, \ldots, X_{n}^{w_{n}}$. We can assume that the $T_{i j}$ 's are w-homogeneous (of respective w-degrees $w_{i} \rho_{i}-\operatorname{deg}_{\mathbf{w}}\left(F_{j}\right)$ ). Then $(i \in\{1, \ldots, n\})$ :

$$
G_{i}=T_{i 1} \cdot F_{1}+\cdots+T_{i n} \cdot F_{n}
$$

is a Generalized Pham System and $\ell_{F}(P)=\ell_{G}(P \cdot \operatorname{det}(T))$.

Remark 1.1. Note that $\operatorname{det}(T)$ is $\mathbf{w}$-homogeneous of $\mathbf{w}$-degree $\sum w_{i} \rho_{i}-\sum \operatorname{deg}_{\mathbf{w}}\left(F_{i}\right)$.

Theorem 1.1 (Weighted version of the Euler-Jacobi Theorem). Let $w$ be a weight vector, $\|w\|=w_{1}+\cdots+w_{n}$, and $F_{1}, \ldots, F_{n}$ with no common solution at the weighted infinity with respect to $\mathbf{w}$. If $P \in \mathbb{K}[\underline{X}]$ and

$$
\operatorname{deg}_{\mathbf{w}}(P)<\sum_{i=1}^{n} \operatorname{deg}_{\mathbf{w}}\left(F_{i}\right)-\|w\|
$$

then we have $\ell_{F}(P)=0$. to $F$ for $\mathbf{w}$.

The integer $\sum_{i} \operatorname{deg}_{\mathrm{w}}\left(F_{i}\right)-\|w\|$ is called the critical number associated

Proof. First, the result is obvious when $F$ is a Generalized Pham System, with multidegree $\delta$, because $\mathbf{w}=\sum \operatorname{deg}_{\mathbf{w}}\left(F_{i}\right)-\|w\|$ is a supporting hyperplane of $\{\delta-1\}+\mathcal{C}(F)$ at $\delta-1$.

Now, if $F$ just defines a zero-dimensional complete intersection, we set $T$ and $G$ as in (3), (4). If $\operatorname{deg}_{\mathbf{w}}(P)<\sum \operatorname{deg}_{\mathbf{w}}\left(F_{i}\right)-\|\mathbf{w}\|$, then $\operatorname{deg}_{\mathbf{w}}(P$. $\operatorname{det}(T))<\sum w_{i} \rho_{i}-\|w\|$ (by Remark 1.1). This last number is exactly the critical number of the system $G$ for $\mathbf{w}$. The theorem follows.

Theorem 1.2 (Weighted version of Macaulay's Theorem). Let $w$ be a weight vector, and $H_{1}, \ldots, H_{n}$ be w-homogeneous polynomials, with no common nontrivial zero. Let $P$ be a $\mathbf{w}$-homogeneous polynomial. If $\operatorname{deg}_{\mathrm{w}}(P)>$ $\sum \operatorname{deg}_{\mathrm{w}}\left(H_{i}\right)-\|w\|$ then $P$ lies in the ideal $\left\langle H_{1}, \ldots, H_{n}\right\rangle$.

Proof. We choose $\rho_{1}, \ldots, \rho_{n}$ and $T$ as in (3). Then, by Example 1.1, $\ell_{H}(P)=\ell_{\left[X_{1}^{\rho_{1}}, \ldots, X_{n}^{\rho_{n}}\right]}(P \cdot \operatorname{det}(T))=0$ as soon as $\mathcal{N D}(P)$ does not meet $\left(\rho_{1}-1, \ldots, \rho_{n}-1\right)-\mathcal{N D}(\operatorname{det}(T))$. This last set is contained in the hyper- 
plane $\mathbf{w}=\sum \operatorname{deg}_{\mathbf{w}}\left(H_{i}\right)-\|w\|$. So if $P$ is homogeneous of degree larger than this number, then $\ell_{H}(P)=0$, and better: $\ell_{H}(P \cdot Q)=0$ for every $Q \in \mathbb{K}[\underline{X}]$. But this implies $P$ lying in the ideal $\left\langle H_{1}, \ldots, H_{n}\right\rangle$, because the global residue operator is dualizing.

Remark 1.2. As noticed by ${ }^{[3]}$ and $^{[5]}$, Macaulay's theorem provides convenient $\rho_{1}, \ldots, \rho_{n}$ for the construction (3), (4): it is sufficient to solve a linear system.

Example 1.3. Let $w=(1,2)$ and

$$
\begin{aligned}
& F_{1}=X_{1}^{2} X_{2}^{2}+X_{1}^{2}+X_{2}^{2}+X_{1} X_{2}+X_{1}+1, \\
& F_{2}=X_{1}^{4}+X_{2}^{2}-X_{1}^{2} X_{2}+X_{1}+1 .
\end{aligned}
$$

Then $F_{1}, F_{2}$ have weighted degrees, with respect to $\mathbf{w}$, repectively 6,4 , and no common solution at the weighted infinity. The system has critical number 7 . So $X_{1}^{8}$ and $X_{2}^{4}$ are in the ideal generated by the $\mathbf{w}$-homogeneous leading parts $X_{1}^{2} X_{2}^{2}$ and $X_{1}^{4}+X_{2}^{2}-X_{1}^{2} X_{2}$. Looking for $a_{1}, a_{2}, b_{1}, b_{2}, b_{3}, u_{1}, u_{2}, v_{1}, v_{2}, v_{3}$ such that:

$$
\begin{aligned}
& X_{1}^{8}=\left(a_{1} X_{1}^{2}+a_{2} X_{2}\right) \cdot X_{1}^{2} X_{2}^{2}+\left(u_{1} X_{1}^{4}+u_{2} X_{1}^{2} X_{2}+b_{3} X_{2}^{2}\right) \cdot\left(X_{1}^{4}+X_{2}^{2}-X_{1}^{2} X_{2}\right) \\
& X_{2}^{4}=\left(u_{1} X_{1}^{2}+u_{2} X_{2}\right) \cdot X_{1}^{2} X_{2}^{2}+\left(v_{1} X_{1}^{4}+v_{2} X_{1}^{2} X_{2}+v_{3} X_{2}^{2}\right) \cdot\left(X_{1}^{4}+X_{2}^{2}-X_{1}^{2} X_{2}\right)
\end{aligned}
$$

we find easily there is a unique solution: $a_{1}=v_{1}=v_{2}=b_{3}=0, a_{2}=u_{1}=$ $-1, b_{1}=b_{2}=u_{2}=v_{3}=1$ and:

$$
T=\left[\begin{array}{cc}
-X_{2} & X_{1}^{4}+X_{1}^{2} X_{2} \\
-X_{1}^{2}+X_{2} & X_{2}^{2}
\end{array}\right]
$$

The Generalized Pham System $G=T \cdot F$ is:

$$
\begin{aligned}
G_{1}= & X_{1}^{8}-X_{2}^{3}-X_{1} X_{2}{ }^{2}-X_{1} X_{2}-X_{2}+X_{1}{ }^{5}+X_{1}{ }^{4}+X_{1}{ }^{3} X_{2} \\
G_{2}= & X_{2}^{4}-X_{1}{ }^{4}-X_{1}{ }^{2} X_{2}{ }^{2}-X_{1}{ }^{3} X_{2}-X_{1}{ }^{3}-X_{1}{ }^{2}+X_{2} X_{1}{ }^{2} \\
& +X_{2}{ }^{3}+2 X_{1} X_{2}{ }^{2}+X_{1} X_{2}+X_{2}+X_{2}{ }^{2} .
\end{aligned}
$$

In Fig. 1, the shaded polytope is $\{\delta-1\}+\mathcal{N} \mathcal{P}\left(G_{1} \cdot G_{2}\right)$, and the shaded polyhedron is $\{\delta-1\}+\mathcal{C}(G)$. The weighted Euler-Jacobi theorem says the $\ell_{G}\left(\mathbf{X}^{\alpha}\right)=0$ for $\alpha$ below the dotted line $\mathbf{w}=13$, but Proposition 1.3 implies this for the cases in the broader white area. 


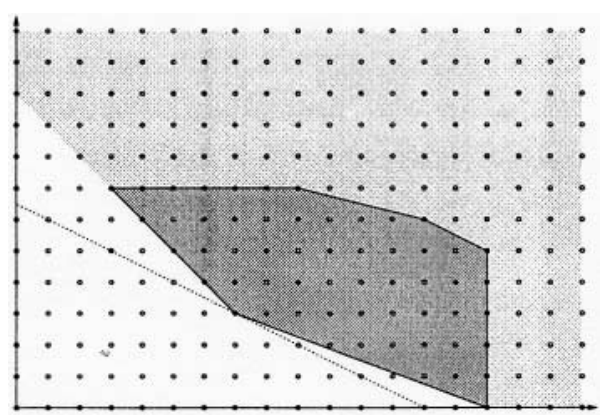

Figure 1. The cone $\{\delta-1\}+\mathcal{C}(G)$ and the Newton polytope $\{\delta-\mathbf{1}\}+$ $\mathcal{N P}\left(G_{1} \cdot G_{2}\right)$

Remark 1.3. The classical Euler-Jacobi Theorem and Macaulay's Theorem are obtained from their weighted versions by choosing for $\mathbf{w}$ the total degree. The weight vector is $(1, \ldots, 1)$. As remarked $\mathrm{in}^{[5]}$, when considering a Generalized Pham System $\left(F_{1}, \ldots, F_{n}\right)$ with multidegree $\delta$, applying the weighted Euler-Jacobi theorem for all weights $\mathbf{w}$ coming from the normal cone of $\mathcal{N P}\left(F_{1} \cdots F_{n}\right)$ at $\delta$ directly implies the vanishing Proposition 1.3.

\section{GENERALIZED NEWTON SUMS AND MULTIVARIATE NEWTON IDENTITIES}

To begin with, let us recapitulate the situation in the univariate case. Let $F=X^{d}+a_{1} X^{d-1}+\cdots+a_{n}$, with roots $\Delta_{1}, \ldots, \Delta_{r}$ (in an algebraic closure of the base field, still assumed to have characteristic zero). Let $\mu_{1}, \ldots, \mu_{r}$ be their respective multiplicities. The Newton Sum with index $j \in \mathbb{N}$ associated to $F$ is:

$$
s_{j}=\sum_{i=1}^{r} \mu_{i} \Delta_{i}^{j} .
$$

The coefficients of $F$ and its Newton Sums are connected by the Newton Identities $\left(s_{0}=n\right)$ :

$$
\begin{aligned}
s_{1} & =-a_{1} \\
s_{2}+a_{1} s_{1} & =-2 a_{2} \\
\vdots & \\
s_{n}+a_{1} s_{n-1}+\cdots+a_{n-1} s_{1} & =-n a_{n} .
\end{aligned}
$$


Note that they make it possible to compute inductively the Newton Sums from the coefficients, and, conversely, the coefficients from the Newton Sums.

The definition of the Newton Sums is generalized to the multivariate case as follows: let $F_{1}, \ldots, F_{n}$ be a complete intersection, with roots $\Delta_{1}, \ldots, \Delta_{r}$ with respective multiplicities $\mu_{1}, \ldots, \mu_{r}$. The Newton Sum with index $\alpha \in \mathbb{N}^{n}$ is:

$$
\mathbf{S}_{\alpha}=\sum_{i=1}^{r} \mu_{i} \mathbf{X}^{\alpha}\left(\Delta_{i}\right)
$$

In other words, the trace of the monomial $\mathbf{X}^{\alpha}$ with respect to $F_{1}, \ldots, F_{n}$.

In order to generalize the univariate Newton Identities to the multivariate case, for Pham Systems, ${ }^{[1]}$ introduces Generalized Newton Sums: objects $\mathbf{S}_{\alpha}$ with $\alpha \in \mathbb{Z}^{n}$, which coincide with the Newton Sums when $\alpha \in \mathbb{N}^{n}$. They set:

$$
\mathbf{S}_{\alpha}=\frac{1}{(2 i \pi)^{n}} \int \frac{\mathbf{X}^{\alpha} \cdot \mathbf{J a c} \cdot d X_{1} \wedge \cdots \wedge d X_{n}}{F_{1} \cdots F_{n}}
$$

for $\alpha \in Z^{n}$, and the integration on a convenient chain. This gives the Newton Sums when $\alpha \in \mathbb{N}^{n}$.

We can set up the algebraic equivalent of this definition for a larger class of systems (containing the Generalized Pham Systems), those verifying:

Condition 1. $X_{1} F_{1}, \ldots, X_{n} F_{n}$ still have finitely many common solutions in $\mathbb{L}^{n}$.

For such systems, we extend the global residue operator $\ell_{F}$ to a linear form $\tilde{\ell}_{F}$ on $\mathbb{K}\left[X_{1}, \frac{1}{X_{1}}, \ldots, X_{n}, \frac{1}{X_{n}}\right]$. It is sufficient to define its value on the Laurent monomials. For $\alpha \in \mathbb{Z}^{n}$,

$$
\tilde{\ell}_{F}\left(\mathbf{X}^{\alpha}\right)=\ell_{\left[X_{1}^{\alpha_{1}^{-}} F_{1}, \ldots, X_{n}^{\alpha_{n}^{-}} F_{n}\right]}\left(\mathbf{X}^{\alpha^{+}}\right)
$$

where $\alpha=\alpha^{+}-\alpha^{-}$, and $\alpha^{+}, \alpha^{-} \in \mathbb{N}^{n}$ (the Transformation Law, 1.1, ensures the consistency of this definition).

Then we define the generalized Newton Sums associated to F:

$$
\mathbf{S}_{\alpha}=\tilde{\ell}_{F}\left(\mathbf{X}^{\alpha} \cdot \mathbf{J a c}\right), \quad \alpha \in \mathbb{Z}^{n} .
$$

Some of the vanishing properties of $\ell$ extend to $\tilde{\ell}$. 


\section{Proposition 2.1.}

1. Suppose $F=\left(F_{1}, \ldots, F_{n}\right)$ defines a zero-dimensional strict complete intersection graded by $\mathbf{w}$. Then for any Laurent Polynomial $P$ such that $\operatorname{deg}_{\mathrm{w}}(P)<\sum \operatorname{deg}_{w}\left(F_{i}\right)-\|w\|$ we have $\tilde{\ell}_{F}(P)=0$.

2. Suppose $F$ is a Generalized Pham System. Then $\tilde{\ell}_{F}(P)=0$ for any Laurent polynomial $P$ such that $\mathcal{N D}(P)$ lies outside $\{\delta-1\}+\mathcal{S}(P)$.

Proof. This immediately comes from the definition of $\tilde{\ell}_{F}$ and Propositions 1.1 and 1.3.

\section{Example 2.1.}

1. Suppose $F$ defines a strict complete intersection, graded by $\mathbf{w}$. Note that $\operatorname{deg}_{\mathbf{w}}(\mathbf{J a c}) \leq \sum \operatorname{deg}_{\mathbf{w}} F_{i}-\|w\|$. So the Generalized Newton Sums $\mathbf{S}_{\alpha}=\tilde{\ell}_{F}\left(\mathbf{X}^{\alpha} \cdot \mathbf{J a c}\right)$ are zero whenever $\mathbf{w}(\alpha)<0$.

2. Suppose $F$ is a Generalized Pham System with multidegree $\delta$ for some term ordering $\preceq$. Let $\mathbf{w}$ be a weight lying inside the normal cone of $\mathcal{N P}\left(F_{1} \cdots F_{n}\right)$ at $\delta$. Then $\{\delta-1\}+\mathcal{C}(F)+$ $(-\mathcal{N} \mathcal{P}(\mathbf{J a c}))$ is included in $\mathcal{C}(G)$. Indeed,

$$
\mathcal{N P}(\mathbf{J a c}) \subset \mathcal{N P}\left(F_{1}, \ldots, F_{n}\right)+\{-1\} \subset\{\delta-1\}+(-\mathcal{C}(G)) .
$$

So the Generalized Newton Sums $\mathbf{S}_{\gamma}$ are zero whenever $\gamma$ is outside $\mathcal{C}(F)$. Specially, $\mathbf{w}=0$ being a supporting hyperplane of $\mathcal{C}(F)$ at 0 , we have $\mathbf{S}_{\gamma}=0$ when $\mathbf{w}(\gamma) \leq 0$, except for $\gamma=0$ : then $\mathbf{S}_{0}=D=d_{1} \cdots d_{n}$.

Example 2.2. Suppose now the $F_{i}$ 's are univariate: $F_{1}=F_{1}\left(X_{1}\right), \ldots, F_{n}=$ $F_{n}\left(X_{n}\right)$. Suppose also that $P$ is reduced modulo $F$. Then $\{\delta-1\}+$ $\mathcal{C}(G)+(-\mathcal{N P}(P)) \subset \mathbb{N}^{n}$.

We state two lemmas from which we will next deduce the Multivariate Newton Identities.

Lemma 2.1. Let $F_{1}, \ldots, F_{n}$ verifying condition 2 . We set:

$$
F_{1} \cdots F_{n}=\sum_{\alpha} b_{\alpha} \mathbf{X}^{\alpha}
$$

Let $P \in \mathbb{K}[\underline{X}]$. We set: $X_{1} \cdots X_{n} \cdot P=\sum_{\alpha} p_{\alpha} \mathbf{X}^{\alpha}$. Then the coefficients of the $F_{i}$ 's, of $P$ and the $\tilde{\ell}_{F}\left(\mathbf{X}^{\alpha} \cdot P\right)$ are connected by the following relations:

$$
\sum_{\alpha} b_{\alpha} \tilde{\ell}_{F}\left(\mathbf{X}^{\alpha-\beta} \cdot P\right)=p_{\beta}
$$

for $\beta \in \mathbb{Z}^{n}$. 
Proof. The proof of the lemma consists in developing $t_{\gamma}=\tilde{\ell}_{F}\left(\mathbf{X}^{\gamma} \cdot P \cdot F_{1}\right.$. $\left.\cdots F_{n}\right)$ in two different ways. First, simplifying by $F_{1} \cdots F_{n}$ with the Transformation Law (1.1), we obtain:

$$
\begin{aligned}
& t_{\gamma}=\ell_{\left[X_{1}^{\gamma_{1}^{-}} F_{1}, \ldots, X_{n}^{\gamma^{\prime n}} F_{n}\right]}\left(\mathbf{X}^{\gamma^{+}} \cdot P \cdot F_{1} \cdots F_{n}\right)=\ell_{\left[X_{1}^{\gamma_{1}^{-}}, \ldots, X_{n}^{\gamma_{n}}\right]}\left(\mathbf{X}^{\gamma^{+}} \cdot P\right) \\
& =\ell_{\left[X_{1}^{\gamma_{1}^{+}+1}, \ldots, X_{n}^{\gamma_{n}+1}\right]}\left(\mathbf{X}^{\gamma^{+}} \cdot X_{1} \cdots X_{n} \cdot P\right) \\
& =\sum_{\alpha} p_{\alpha} \ell_{\left[X_{1}^{\gamma_{1}^{+}+1}, \ldots, X_{n}^{\gamma_{n}^{\prime n}+1}\right]}\left(\mathbf{X}^{\gamma^{+}+\alpha}\right)=p_{-\gamma}
\end{aligned}
$$

by using the arguments in Example 1.1. On the other hand, developing the product $F_{1} \cdots F_{n}$ yields:

$$
t_{\gamma}=\sum_{\alpha} b_{\alpha} \tilde{\ell}_{F}\left(\mathbf{X}^{\gamma+\alpha} \cdot P\right)
$$

Now set $\beta=-\gamma$.

Next we present a technical lemma ${ }^{[8]}$ which provides a very useful way of representing the jacobian of the polynomials $F_{1}, \ldots, F_{n}$. First we set, for $i=1, \ldots, n$ :

$$
F_{i}=\sum_{\alpha} a_{\alpha}^{(i)} \mathbf{X}^{\alpha}
$$

Lemma 2.2. Let $\mathbf{J a c}$ be the jacobian of the polynomials $F_{1}, \ldots, F_{n}$ and for any family $\alpha^{1}, \ldots, \alpha^{n}$ of multi-indices in $Z^{n}$ let us denote by $\Gamma_{\left[\alpha^{1}, \ldots, \alpha^{n}\right]}$ the determinant of the matrix $\left(\alpha_{j}^{i}\right)_{i, j}$. Then the following equality holds:

$$
X_{1} \cdots X_{n} \cdot \mathbf{J a c}=\sum_{\alpha^{1}, \cdots, \alpha^{n}} a_{\alpha^{1}}^{(1)} \cdots \cdot a_{\alpha^{n}}^{(n)} \cdot \Gamma_{\left[\alpha^{1}, \ldots, \alpha^{n}\right]} \cdot \mathbf{X}^{\alpha^{1}} \cdots \cdots \mathbf{X}^{\alpha^{n}} .
$$

Proof. The way of representing the polynomials $F_{j}$ allows us to write:

$$
X_{i} \cdot \frac{\partial F_{j}}{\partial X_{i}}=X_{i} \cdot\left(\sum_{\|\alpha\| \leq d_{j}} a_{\alpha}^{(j)} \frac{\partial \mathbf{X}^{\alpha}}{\partial X_{i}}\right)=\sum_{\|\alpha\| \leq d_{j}} a_{\alpha}^{(j)} \alpha_{i} \mathbf{X}^{\alpha}
$$

and thus: 


$$
X_{1} \cdots X_{n} \cdot \mathbf{J a c}=\left|\begin{array}{ccc}
X_{1} \cdot \frac{\partial F_{1}}{\partial X_{1}} & \ldots & X_{n} \cdot \frac{\partial F_{1}}{\partial X_{n}} \\
\vdots & & \vdots \\
X_{1} \cdot \frac{\partial F_{n}}{\partial X_{1}} & \ldots & X_{n} \cdot \frac{\partial F_{n}}{\partial X_{n}}
\end{array}\right|
$$

Replacing in this determinant the equalities in (7), the desired result is obtained merely by developing the determinant.

Theorem 2.1 (Multivariate Newton Identities). Let $\beta \in Z^{n}$ a multi-index. Then:

$$
\sum_{\alpha^{1}, \ldots, \alpha^{n}} a_{\alpha^{1}}^{(1)} \cdots \cdots a_{\alpha^{n}}^{(n)} \cdot \mathbf{S}_{\alpha^{1}+\cdots+\alpha^{n}-\beta}=\sum_{\alpha^{1}+\cdots+\alpha^{n}=\beta} \Gamma_{\left[\alpha^{1}, \ldots, \alpha^{n}\right]} \cdot a_{\alpha^{1}}^{(1)} \cdots \cdots a_{\alpha^{n}}^{(n)} .
$$

Proof. These identities come directly from Lemma 2.1 with $P=\mathbf{J a c}$ and from Lemma 2.2.

Now we raise the problem of knowing when the Multivariate Newton Identities make possible the computation of the Newton Sums from the coefficients. A good strategy consists in choosing a weight vector $w \in \mathbb{N}^{n}$ to organize the computations: we demand the leading w-homogeneous part of the product $F_{1} \cdots F_{n}$ be a monomial $\mathbf{X}^{\delta}$ with weight larger than that of any other monomial in $F_{1} \cdots F_{n}$, so that the formulas, expressing $\mathbf{S}_{\delta-\beta}$ in terms of the $\mathbf{S}_{\alpha-\beta}$ for $\mathbf{w}(\alpha)<\mathbf{w}(\delta)$, become recurrent. This requires that the $F_{i}$ 's have all the same shape, that is: a monomial plus lower terms (with respect to w). We can also assume that the leading monomials have coefficient 1 . Our final need is to have initial conditions. They are given by the extension of the weighted Euler-Jacobi Theorem to $\tilde{\ell}$ (Example 2.1,1). However, using this result requires that $\left(F_{1}, \ldots, F_{n}\right)$ has no zero at the weighted infinity.

Finally, the systems $F$ which fulfill all of our requirements, those for which we know how to use the Newton Identities, are exactly the Generalized Pham System. In this case, the Newton Identities are as follows:

$$
\begin{gathered}
\mathbf{S}_{\delta-\beta}+\sum_{\mathbf{w}(\beta)<\mathbf{w}\left(\sum_{i} \alpha^{i}\right)<\mathbf{w}(\delta)} a_{\alpha^{1}}^{(1)} \cdots \cdots a_{\alpha^{n}}^{(n)} \cdot \mathbf{S}_{\alpha^{1}+\cdots+\alpha^{n}-\beta} \\
=\sum_{\alpha^{1}+\cdots+\alpha^{n}=\beta}\left(\Gamma_{\left[\alpha^{1}, \cdots, \alpha^{n}\right]}-D\right) \cdot a_{\alpha^{1}}^{(1)} \cdots \cdots a_{\alpha^{n}}^{(n)}
\end{gathered}
$$

where $D=d_{1} \cdots d_{n}, \beta \in Z^{n}$. Indeed, $\mathbf{S}_{0}=$ Trace(1) equals the dimension of the quotient algebra $\mathcal{A}$, which is $d_{1} \cdots d_{n}$, and all of the other Newton sums $\mathbf{S}_{\alpha}$ with $\mathbf{w}(\alpha) \leq 0$ are zero, by Example 2.1, 2 . 


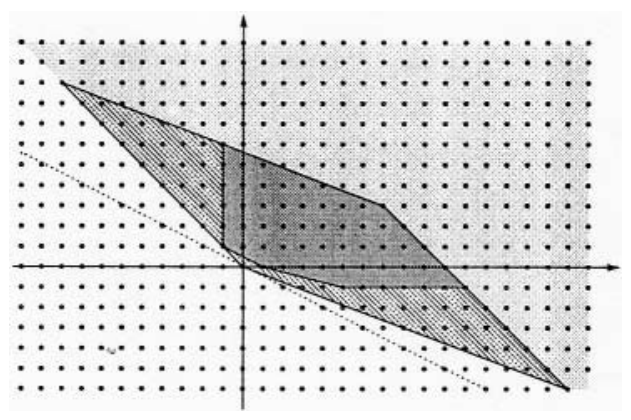

Figure 2. Computing the Newton Sum $\mathbf{S}_{(7,3)}$.

Example 2.3. By applying the Newton Identity $\mathbf{S}_{7.3}+\cdots$ for the system $\left(G_{1}, G_{2}\right)$ of Example 1.2, we express $\mathbf{S}_{7,3}$ in terms of the Generalized Newton sums whose indices lie inside the dark polytope in Fig. 2. To express $\mathbf{S}_{7,3}$ in function of the coefficients of the system we just need to apply all the identities $\mathbf{S}_{\alpha}+\cdots$ for $\alpha$ in the union of the striped area and the dark polygon, since all $\mathbf{S}_{\alpha}$ for $\alpha$ in the white area, are zero.

Precisely, the considered Newton Identity is:

$$
\begin{aligned}
& \mathbf{S}_{7,3}-\mathbf{S}_{-1,6}+\mathbf{S}_{7,2}-\mathbf{S}_{9,1}-\mathbf{S}_{10,0}+\mathbf{S}_{2,4}-\mathbf{S}_{0,5}+\mathbf{S}_{4,3}+2 \mathbf{S}_{8,1}-\mathbf{S}_{10,-1} \\
& \quad+\mathbf{S}_{9,0}+\mathbf{S}_{1,4}-\mathbf{S}_{-1,5}+\mathbf{S}_{3,3}+\mathbf{S}_{7,1}-\mathbf{S}_{11,-1}+\mathbf{S}_{8,0}-4 \mathbf{S}_{0,4}+3 \mathbf{S}_{2,3} \\
& \quad-\mathbf{S}_{6,1}+5 \mathbf{S}_{3,2}-2 \mathbf{S}_{-1,4}-3 \mathbf{S}_{1,3}-\mathbf{S}_{9,-1}-\mathbf{S}_{6,0}+2 \mathbf{S}_{2,2}-3 \mathbf{S}_{0,3}+5 \mathbf{S}_{4,1} \\
& \quad-\mathbf{S}_{8,-1}+\mathbf{S}_{5,0}-\mathbf{S}_{1,2}-2 \mathbf{S}_{-1,3}+4 \mathbf{S}_{3,1}-2 \mathbf{S}_{7,-1}-4 \mathbf{S}_{0,2}+2 \mathbf{S}_{2,1} \\
& \quad-2 \mathbf{S}_{6,-1}+3 \mathbf{S}_{3,0}-\mathbf{S}_{-1,2}-2 \mathbf{S}_{1,1}+2 \mathbf{S}_{2,0}-2 \mathbf{S}_{0,1}+\mathbf{S}_{1,0}-\mathbf{S}_{-1,1}=0 .
\end{aligned}
$$

Remark 2.1. When considering the case $n=1$, which is a univariate polynomial, the above Newton Identities are exactly the classical Newton Identities presented in (5).

\section{GENERATING FUNCTIONS FOR THE NEWTON SUMS}

With the notations of Lemma 2.1, we had:

$$
\sum_{\alpha} b_{\alpha} \tilde{\ell}_{F}\left(\mathbf{X}^{\alpha+\gamma} \cdot P\right)=p_{-\gamma}, \quad \gamma \in Z^{n} .
$$

Multiplying both sides of the equality by $1 / \mathbf{X}^{\gamma}$, and then summing for all $\gamma \in$ $Z_{s}^{n}$ yields, after reordering properly the terms: 


$$
F_{1} \cdots F_{n} \cdot \sum_{\gamma \in Z^{n}} \frac{\tilde{\ell}_{F}\left(\mathbf{X}^{\gamma} \cdot P\right)}{\mathbf{X}^{\gamma}}=P \cdot X_{1} \cdots X_{n} .
$$

At this point we guess this may lead to generating functions for the family of the $\tilde{\ell}_{F}\left(\mathbf{X}^{\gamma} \cdot P\right), \gamma \in Z^{n}$, and especially for the Generalized Newton Sums, with $P=$ Jac. The following lemma will give the appropriate setting for these considerations. Let us introduce the necessary notions: let $\mathbf{w}$ be a weight and $\mathbb{B}_{\mathbf{w}}$ be the set of all expressions $\sum_{\gamma \in Z^{n}} \frac{u_{\gamma}}{\mathbf{X}^{\eta}}$ with $u_{\gamma} \in \mathbb{K}$, and such that

1. for any $k \in \mathbb{Z}$, all but a finite number of the $u_{\gamma}$ with $w(\gamma)=k$ are zero, and

2. there exists $k_{0} \in Z$ such that $u_{\gamma}=0$ as soon as $w(\gamma)<k_{0}$.

This is naturally a graded $\mathbb{K}$-algebra. If $P$ is a polynomial whose whomogeneous leading part is a monomial, so $P=\mathbf{X}^{\delta}+R$ with $\mathbf{w}(R)<\mathbf{w}(\delta)$, then the formal series

$$
S=\sum_{i \in \mathbb{N}}\left(\frac{R}{\mathbf{X}^{\delta}}\right)^{i}
$$

is well-defined in $\mathbb{B}_{\mathbf{w}}$, and $P \cdot S=1$. In this way in our ring $\mathbb{B}_{\mathbf{w}}$ all the inverses of such polynomials $P$ are represented. We say that $S$ is the series expansion of $1 / P$ in $\mathbb{B}_{\mathbf{w}}$.

Remark 3.1. If $\mathbb{K} \subset \mathbb{C}$, we define:

$$
\text { Log : } \begin{array}{ccc}
\mathbb{C}^{n} & \rightarrow & \mathbb{R}^{n} \\
\left(z_{1}, \ldots, z_{n}\right) & \mapsto & \left(\log \left|z_{1}\right|, \ldots, \log \left|z_{n}\right|\right)
\end{array}
$$

and $N_{\delta}$ as the normal cone of $P$ at $\delta$. Then the series $S$ converges in $\log ^{-1}\left(b+N_{\delta}\right)$ for some $b \in \mathbb{R}^{n}\left(\operatorname{see}^{[7]}\right)$.

Lemma 3.1. Let $F_{1}, \ldots, F_{n}$ be a Generalized Pham System, with multidegree $\delta$. With the notations of Lemma 2.1: the series

$$
\sum_{\gamma \in \mathbb{Z}^{n}} \frac{\tilde{\ell}_{F}\left(\mathbf{X}^{\gamma} \cdot P\right)}{\mathbf{X}^{\gamma}}
$$

lies in $\mathbb{B}_{\mathbf{w}}$, for any weight $\mathbf{w}$ lying inside the normal cone of $\mathcal{N P}\left(F_{1} \cdots F_{n}\right)$ at $\delta$, and:

$$
\sum_{\gamma \in Z^{n}} \frac{\tilde{\ell}_{F}\left(\mathbf{X}^{\gamma} \cdot P\right)}{\mathbf{X}^{\gamma}}=P \cdot \frac{X_{1} \cdots X_{n}}{F_{1} \cdots F_{n}}
$$

in $\mathbb{B}_{\mathbf{w}}$. 
Proof. The series is in $\mathbb{B}_{\mathbf{w}}$ because of Proposition 2.1, 2. Its expression as a rational fraction comes from the above calculation.

Suppose we have a zero-dimensional system $F=\left(F_{1}, \ldots, F_{n}\right)$, which can be transformed into a Generalized Pham System $G(1 \leq i \leq n)$ :

$$
G_{i}=T_{i 1} \cdot F_{1}+\cdots+T_{i n} \cdot F_{n}
$$

For $\gamma \in \mathbb{N}^{n}: \ell_{F}\left(\mathbf{X}^{\gamma} \cdot P\right)=\ell_{G}\left(\mathbf{X}^{\gamma} P \operatorname{det}(T)\right)$. So:

$$
\sum_{\gamma \in \mathbb{N}^{n}} \frac{\ell_{F}\left(\mathbf{X}^{\gamma} \cdot P\right)}{\mathbf{X}^{\gamma}}=P \cdot \operatorname{det}(T) \frac{X_{1} \cdots X_{n}}{G_{1} \cdots G_{n}}+\sum_{\gamma \notin \mathbb{N}^{n}} \frac{m_{\gamma}}{\mathbf{X}^{\gamma}}
$$

for some $m_{\gamma}$. Precisely, $m_{\gamma}=\tilde{\ell}_{G}\left(\mathbf{X}^{\gamma} \cdot P \cdot \operatorname{det}(T)\right)$.

Example 3.1. If $F$ defines a zero-dimensional strict complete intersection, it can be transformed as above into a Generalized Pham System, with the method used in the Proof of 1.3, and Macaulay's Theorem (see Remark 1.2). This was pointed out $\mathrm{in}^{[2]}$ and ${ }^{[5]}$. In these papers the equality (15) was used to compute the residues by series expansions.

Example 3.2. For $F$ defining a zero-dimensional complete intersection, there always exist "eliminating equations":

$$
T_{i 1} \cdot F_{1}+\cdots+T_{i n} \cdot F_{n}=\chi_{i}\left(X_{i}\right)
$$

for $i=1 \ldots n$, with $T_{i j} \in \mathbb{K}[\underline{X}]$ and the $\chi_{i} \in \mathbb{K}\left[X_{i}\right]$ non-zero. We get:

$$
\sum_{\alpha \in \mathbb{N}^{n}} \frac{\mathbf{S}_{\alpha}}{\mathbf{X}^{\alpha}}=\operatorname{det}(T) \cdot \mathbf{J a c} \cdot \frac{X_{1} \cdots X_{n}}{\chi_{1} \cdots \chi_{n}}+\sum_{\alpha \notin \mathbb{N}^{n}} \frac{m_{\alpha}}{\mathbf{X}^{\alpha}}
$$

for some $m_{\alpha}, \alpha \notin \mathbb{N}^{n}$. This result, for $n=2$, had been established by Jacobi in 1835 in the particular case when every $\chi_{i}$ is the Eliminant polynomial of $F$ for $X_{i}$ (that is: $\chi_{i}$ has no multiple root, and its roots are precisely the $X_{i^{-}}$ coordinates of the roots of $F)^{[9]}$. Much later, in 1991, P. Pedersen proved the general case ${ }^{[11]}$.

Now we find what are precisely the generating series for the Newton Sums (without terms $m_{\alpha}$ with $\alpha \notin \mathbb{N}^{n}$ ), and for the families defined in a similar way, that is: the families of all of the $\lambda\left(\mathbf{X}^{\alpha}\right)$ for a given linear form $\lambda$ on $\mathcal{A}$. Note that, since $\ell_{F}$ is dualizing, there exists a polynomial $P_{\lambda}$ (unique modulo $\left.\left\langle F_{1}, \ldots, F_{n}\right\rangle\right)$ such that $\lambda: Q \mapsto \ell_{F}\left(Q \cdot P_{\lambda}\right)$. 
Proposition 3.1. Suppose $F_{1}, \ldots, F_{n}$ define a zero-dimensional complete intersection. Let $f$ by any $\mathbb{K}$-linear form on $A$. Then the generating function of the $f\left(X^{\alpha}\right)$ is obtained by series expansion in $1 / X_{1}, \ldots, 1 / X_{n}$ of a rational function:

$$
\sum_{\alpha \in \mathbb{N}^{n}} \frac{f\left(X^{\alpha}\right)}{\mathbf{X}^{\alpha}}=\frac{X_{1} \cdots X_{n}}{\chi_{1}\left(X_{1}\right) \cdots \cdot \chi_{n}\left(X_{n}\right)} \cdot N F_{\chi}\left(\operatorname{det}(T) \cdot P_{\lambda}\right)
$$

where $T$ is a transformation matrix of $F$ into $\chi$ (as in 16) and $N F_{\chi}$ gives the normal form with respect to $\chi_{1}, \ldots, \chi_{n}$.

Proof. For $\alpha \in \mathbb{N}^{n}: \ell_{\chi}\left(\mathbf{X}^{\alpha} \cdot N F_{\chi}\left(\operatorname{det}(T) \cdot P_{f}\right)\right)=\ell_{\chi}\left(\mathbf{X}^{\alpha} \cdot \operatorname{det}(T) \cdot P_{f}\right)=f\left(\mathbf{X}^{\alpha}\right)$. And for $\alpha \notin \mathbb{N}^{n}$ :

$$
\ell_{\chi}\left(\mathbf{X}^{\alpha} \cdot N F_{\chi}\left(\operatorname{det}(T) \cdot P_{f}\right)\right)=0
$$

because the $\chi_{i}^{\prime}$ 's are univariate (see Example 2.2).

Example 3.3. This formula produces some well-known identities for a single univariate polynomial $P(X)$ :

- With $f=$ Trace, the $f\left(X^{i}\right)$ are the Newton Sums $s_{i}$ associated to $P$, and

$$
\sum_{i \in \mathbb{N}} \frac{s_{i}}{X^{i}}=\frac{X P^{\prime}}{P}
$$

- With $f=\ell_{P}$, the global residue operator:

$$
\sum_{i \in \mathbb{N}} \frac{\ell_{P}\left(x^{i}\right)}{X^{i}}=\frac{X}{P}
$$

\section{AN APPLICATION: PHAM SYSTEMS OF DEGREE 2}

In the particular case when all the $F_{i}$ have degree 2 and the system is a Pham system (then we denote: $F_{i}=X_{i}^{2}+\sum_{j=1}^{n} u_{i, j} X_{j}+v_{i}, i=1, \ldots, n$ ), the Newton Sums $\mathbf{S}_{1,1, \ldots, 1,0,0, \ldots, 0}$ have quite a simple expression as functions of the coefficients.

Indeed, for such a system, developing in Laurent series the function

$$
\frac{X_{1} \cdots X_{n} \cdot \mathbf{J a c}}{F_{1} \cdots F_{n}}
$$


gives:

$$
\mathbf{S}_{\alpha}=\sum_{M, I, J}(-1)^{|M|} \cdot 2^{\operatorname{Card} I} \cdot \prod_{i=1}^{n}\left(\begin{array}{c}
m_{i, 0}+\cdots+m_{i, n} \\
m_{i, 0} \ldots, m_{i, n}
\end{array}\right) \cdot \operatorname{det} U(J) \cdot \prod_{i=1}^{n} v_{i}^{m_{i, 0}} \cdot \prod_{i, j=1}^{n} u_{i, j}^{m_{i, j}}
$$

where the sum is carried over all of the decompositions $I \uplus J=\{1, \ldots, n\}$ and matrices $M=\left(m_{i, j}\right), i=1, \ldots, n, j=0, \ldots, n$ with positive entries, such that:

$$
\alpha_{i}=\sum_{j=0, \ldots, n} m_{l, j}-\sum_{i=1, \ldots, n} m_{i, l}-\chi_{I}(i), \quad i=1, \ldots, n
$$

where $\chi_{I}(i)=1$ if $i \in I, 0$ otherwise. Our notation $U(J)$ stands for the submatrix of the $u_{i, j}$ with $i \in J, j \in J$.

For $\alpha=(1, \ldots, 1,0, \ldots, 0): k$ times 1 followed by $n-k$ times 0 , these $I, J, M$ should verify:

$$
2 \sum_{j=0, \ldots, n} m_{l, j}-\sum_{i=1, \ldots, n} m_{i, l}= \begin{cases}1 & \text { if } i \leq k \text { and } \quad i \in I \\ -1 & \text { if } i>k \text { and } \quad i \in J . \\ 0 & \text { otherwise }\end{cases}
$$

Adding these equalities together one gets:

$$
|M|+\sum_{i=1}^{n} m_{i, 0}=\operatorname{Card}(\{1, \ldots, k\} \cap I)-\operatorname{Card}(\{k+1, \ldots, n\} \cap J) .
$$

But obviously everytime $i \leq k$ and $i \in I$ then the $i$-th line of $M$ has a nonzero entry, so $|M| \geq \operatorname{Card}(\{1, \ldots, k\} \cap I)$. From this we deduce that for all $i$, $m_{i, 0}=0 ; J \subset\{1, \ldots, k\}$; and finally that the matrix of the $m_{i, j}, i \in I, j \in I$ is a permutation matrix (all coefficients equal to 0 except precisely one 1 on each column and one 1 on each row) and the remaining coefficients $m_{i, j}=0$ for $i \notin I$ or $j \notin I$. This Newton Sum has an expression of the following type:

$$
\begin{aligned}
\mathbf{S}_{1, \ldots, 1,0 \ldots, 0} & =2^{n-k} \cdot \sum_{J \uplus L=\{1, \ldots, k\}, \tau \in \Sigma_{L}}(-2)^{\operatorname{Card}(L)} \cdot \operatorname{det}(U(J)) \cdot \prod_{i \in L} u_{i, \tau(i)} \\
& =(-1)^{k} 2^{n-k} \sum_{\sigma \in \Sigma_{k}} t(\sigma) \prod_{i=1}^{k} u_{i, \sigma(i)}
\end{aligned}
$$

where $t(\sigma)=\left(2^{l_{1}}-1\right) \cdots\left(2^{l_{p}}-1\right)$ when $\sigma$ decomposes as a product of $p$ cycles with disjoint supports of respective lengths $l_{1}, \ldots, l_{p}$. 


\section{ACKNOWLEDGMENTS}

We thank E. Becker for his comments and support for this work since he proposed to us the question about the existence of generating functions for the Multivariate Newton Sums and other kinds of similar multivariate symmetric functions. Partially supported by the grant DGESIC PB 98-0713C02-02 (Ministerio de Educación y Cultura).

\section{REFERENCES}

1. Aizenberg, L.A.; Kytmanov, A.M. Multidimensional Analogues of Newton's Formulas for Systems of Nonlinear Algebraic Equations and some of their Applications. Sibirskii Matematicheskii Zhurnal 1981, 22 (2), 19-39. English translation in Siberian Mathematical J. 1981, 22, 180-189.

2. Aizenberg, L.A.; Yuzhakov, A. Integral Representations and Residues in Multidimensional Complex Analysis; Transactions of Mathematical Monographs 58, American Mathematical Society, 1983.

3. Aizenberg, L.A.; Tsikh, A.K. Application of Multidimensional Logarithmic Residue to Systems of Nonlinear Algebraic Equations. Sibirskii Matematicheskii Zhurnal 1979, 20 (4), 699-707. English Translation in Siberian Mathematical J. 1980, 20, 485-491.

4. Becker, E.; Cardinal, J.-P.; Roy, M.-F.; Szafraniec, Z. Multivariate Bezoutians, Kronecker Symbol and Eisenbud-Levine Formula. In Algorithms in Algebraic Geometry and Applications; Progress in Mathematics; Birkhäuser: 1996; Vol. 143, 79-104.

5. Cattani, E.; Dickenstein, A.; Sturmfels, B. Computing Multidimensional Residues. In Algorithms in Algebraic Geometry and Applications; Progress in Mathematics; Birkhäuser: 1996; Vol. 143, 180189.

6. Cardinal, J.-P.; Mourrain, B. Algebraic Approach of Residues and Applications. In The Mathematics of Numerical Analysis; Lectures in Applied Mathematics; American Mathematical Society: 1996; Vol. 32, 189-210.

7. Gelfand, I.M.; Kapranov, M.; Zelevinsky, A. Discriminants, Resultants and Multidimensional Determinants; Birkhäuser, 1994.

8. Gonzalez-Lopez, M.-J.; Gonzalez-Vega, L. Newton Identities in the Multivariate Case: Pham Systems. In Gröbner Bases and Applications; London Mathematical Society Lecture Notes Series; Cambridge University Press, 1998; Vol. 251, 351-366. 
9. Jacobi, C. Theoremata Nova Algebrica Systema Duarum Aequationum Inter Duas Cariabiles Propositarum. J. Reine Anweg. Math. 1835, 14, 281-288.

10. Kreuzer, M.; Kunz, E. Trace in Strict Frobenious Algebras and Strict Complete Intersections. J. Reine Anweg. Math. 1987, 381, 181-204.

11. Pedersen, P. Calculating Multidimensional Symmetric Functions using Jacobi's Formula. In Applied Algebra, Algebraic Algorithms and Error-correcting Codes; Lecture Notes in Computer Science; Springer Verlag; 1991; Vol. 539, 304-317.

12. Roy, M.-F. Basic Algorithms in Real Algebraic Geometry: from Sturm Theorem to the Existential Theory of Reals. In Lectures on Real Geometry in Memoriam of Mario Raimondo; Expositions in Mathematics; de Gruyter; 1996; Vol. 23, 1-67.

13. Roy, M.-F.; Spirzglas, A. Bezoutiens et Residus, Affines, Projectifs et dans le Tore. Preprint, 2000.

14. Tsikh, A.K. Multidimensional Residues and their Applications. Translation of Mathematical Monographs, Vol. 103, American Mathematical Society, 1992. 